\title{
Umckaloabo ${ }^{\circledR}$
} und die Sicherheit von Phytopharmaka

Winterzeit ist Erkältungszeit, Husten, Schnupfen, Heiserkeit \& Co. erwarten uns. Erwachsene leiden im Mittel 2- bis 5-mal im Jahr, Kinder sogar noch häufiger an Infekten der Atemwege. Bei niedergelassenen Ärzten machen sie zwei Drittel aller zu behandelnden Infektionen aus. Patienten, die wegen damit verbundener Beschwerden in die Praxis oder in die Apotheke kommen, wollen vor allem eines: etwas tun, um rasch wieder gesund zu werden. Wer kann es sich schon leisten, krank zu sein! Gut so! Der Wille, aktiv zur eigenen Genesung beizutragen, trägt offenbar auch bei »Erkältungen» Früchte: 476 Patienten mit akuten Atemwegsinfekten wurden in einer randomisierten Studie untersucht (es ging um den Nutzen von Antibiotika). Das Einzige, was statistisch signifikant nützte, war das persönliche Bekenntnis des Patienten zur gewählten Behandlung (was gar nicht half, waren die Antibiotika [1]).

Wer kämpft, kommt schneller wieder hoch, wer sich hängen lässt, verliert. Das gilt auch, wenn es im Winter im Halse kratzt und die Nase zu laufen beginnt. Natürlich müssen die Mittel angemessen sein. Nil nocere! Pflanzliche Arzneien treffen hier das rechte Maß und viele Patienten entscheiden sich spontan dafür. Dass der Nutzen der Phytopharmaka dennoch nicht allein mit dem Patientenwillen zu begründen ist (wie manche Kritiker es gern im negativen Sinne darstellen), belegen nicht weniger als sieben Beiträge im vorliegenden Heft dieser Zeitschrift. Einer davon berichtet über Anwendungsergebnisse mit einem "Klassiker» der Kommission E, einer Kombination von Primula veris und Thymus vulgaris. Bei den sechs anderen geht es um eine europäische Weiterentwicklung aus der afrikanischen Volksmedizin, einen Extrakt aus der Wurzeldroge von Pelargonium sidoides. Zwei große klinische Studien in der vom BfArM neu zugelassenen Indikation "akute Bronchitis« wurden kürzlich schon in dieser Zeitschrift referiert. Die Reviews von zwei weiteren Therapiestudien kommen in dieser Ausgabe dazu. Drei Originala analysieren darüber hinaus mit pharmakologischen Ergebnissen in vitro und am Tier Ansätze zur Aufklärung des Wirkmechanismus.
Ein 4. Originalbeitrag gibt eine aktuelle Übersicht über die Stoffgruppe der Cumarine in Verbindung mit mutmaßlichen Risiken des Wurzelextraktes aus Pelargonium sidoides. Solche Darstellungen waren bisher in dieser Zeitschrift eher selten. Vielleicht sollten wir uns in Zukunft mehr daran gewöhnen. Eine tragende Säule für die Sicherheit pflanzlicher Arzneimittel ist zwar nach wie vor auch deren lange Anwendungstradition. Herstellern ist dennoch zu empfehlen, Untersuchungen zur Unbedenklichkeit in Zukunft den gleichen Stellenwert einzuräumen, wie den Nachweisen zur Wirksamkeit. Therapierisiken, seien sie reell oder nur hypothetisch, sind auch ein Schwert im Wettbewerb. Arzneimittel der besonderen Therapierichtungen - die Phytotherapie zählt dazu - sind ihrer gesetzlichen Bestimmung nach nicht nur als Konkurrenz gegenüber konventioneller Arznei gedacht, sondern auch eine Herausforderung für das zugehörige akademische Lager. Wer Heilung durch Arznei nicht allein als WirkstoffPhänomen versteht, sondern das Wechselspiel zwischen Patient und Umfeld in die Nutzenbemessung einbezieht, kommt damit schnell mit altgehegten Grundsätzen der Pharmakologie in Kollision!

Mit Gegenbewegungen sollte jeder rechnen, dessen pflanzliche Arznei besonderen Erfolg am Markt hat. In diesem Sinne wurde zum Beispiel in der Ausgabe 3/2003 der Zeitschrift arznei-telegramm (at) beklagt, dass ein Handelspräparat aus Pelargonium sidoides mit jährlich 4 Millionen verkauften Packungen zu den "Schnelldrehern« der Apotheke zähle. Weil nicht sein konnte, was nicht sein durfte, wurde auch gleich ein Lösungsvorschlag für die Behörde mit angehängt: "Ob die noch ausstehende Nachzulassung dem Mittel ein Ende bereitet? « Dieses Ende blieb aus. Vielmehr wurde dem Präparat aufgrund vorgelegter Studien zur Wirksamkeit und Unbedenklichkeit im Dezember 2005 vom BfArM die Nachzulassung erteilt. Nun fiel dem at eine mutmaßliche Hepatotoxizität des Präparates auf: „Von mindestens 78 Meldungen, die dem BfArM in Verbindung mit Pelargonium sidoides seit 1990 vorliegen, betreffen zwei die Leber und werden in der Kausalität von der Behörde als ,mindestens möglich’ eingestuft« heißt 


\section{Editorial}

es dazu im Heft 6/2006. Ein Jahr später wandelt sich der Fokus auf allergische Reaktionen: „15 Berichte sprechen für zum Teil bedrohliche akute Typ-1Überempfindlichkeitsreaktionen« hieß es zuletzt im at $\mathrm{Nr}$. 12/2007. Immerhin wurde diesmal sogar ein nachprüfbares Literaturzitat dafür genannt. Daraus geht unter anderem hervor, dass von den 15 »bedrohlichen« Fällen nur einer von einem Arzt gesehen worden war!

Um nicht missverstanden zu werden: Spontanmeldungen von Apothekern (und Ärzten) sind im Rahmen der Pharmakovigilanz das wichtigste Frühwarnsystem für seltenere Risiken. Wer immer sich der Mühe unterzieht, einen Verdachtsfall zu melden, verdient dafür Dank und Anerkennung. Unsere bereits in einem früheren Editorial (2) zum Ausdruck gebrachte Kritik betrifft nicht die Meldungen, sondern deren Bewertung. Von dem hier genannten Pelargonium-sidoides-Präparat wurden von 1994 bis 2006 etwa 304 Millionen »definierte Tagesdosen» (DDD) verkauft. Mit dieser Zahl sind die beiden (Zufalls-?)Meldungen über angestiegene Leberenzyme zu relativieren. An verbrauchsbezogenen Schätzungen des relativen Risikos, wie die Praktiker sie bräuchten, fehlt es jedoch in unserem Überwachungssystem. Anstelle des quantitativen Gruppenvergleichs tritt die qualitative Bewertung des Einzelfalls, wofür es aber weder allgemeingültige Regeln noch validierte Methoden gibt. Die Auslegung sol- cher Berichte ist in hohem Maße dem Ermessen des Einzelnen überlassen. Darin liegt auch eine Einladung für Interessensvertreter, die pflanzliche Arzneimittel lieber heute als morgen aus dem Markt genommen sähen.

Forschen und darüber berichten ist auf Dauer sicherlich die wichtigste und wirksamste Maßnahme zur Gewährleistung der Sicherheit von Phytopharmaka. Die Hersteller pflanzlicher Arzneimittel werden in Zukunft mehr für die Dokumentation der Sicherheit ihrer Produkte aufwenden müssen. Nach den Erfahrungen der letzten Jahre besteht aber auch begründeter Anlass für die Fachverbände der Phytotherapie, sich engagiert missbräuchlicher Risikopropaganda entgegenzustellen, vor der kein erfolgreicher Hersteller mehr gefeit ist.

Volker Schulz, Berlin

\section{LITERATUR}

1 Little P, Williamson I, Warner G, Gould C, Gantley M, Kinmonth Al: Open randomised trial of prescribing strategies in managing sore throat. BMJ 1997; 314: 722-727.

2 Schulz V: Pharmakovigilanz in der Phytotherapie: Ja zur Vorsorge, nein zum Missbrauch [Editorial]. Z Phytotherapie 2006; 27: 159-160. 